Basic Health Sciences

Poster

Abstract ID: 89

\title{
Piper sarmentosum induced apoptosis via mitochondrial pathway in human lung adenocarcinoma cells, A549
}

\author{
Azila Sirajudeen | Aisyah Hanani Mohd Tahir | Radiah Abdul Ghani \\ Department of Biomedical Science, Kulliyyah of Allied Health Sciences, International Islamic \\ University Malaysia
}

Introduction: Lung cancer has been reported as one of the most common types of cancer worldwide. Current cancer treatments like chemotherapy do not result in a complete cure and are known to cause side effects in the patients. Therefore, alternative treatment strategies are being explored, one of which is to investigate the potential of the local herbs in this regard. Piper sarmentosum (daun kaduk) has received much attention due to its anti-cancer properties in A549 cells. In this study, the cell cycle profile and mechanisms of cell death induced by $P$. sarmentosum were investigated using a flow cytometer. Methods: The cell cycle profile changes were observed using propidium iodide staining while the type of cell death was analyzed using Annexin-V assay. Caspases $-3 / 7,8$ and 9 and cytochrome $c$ assays were elucidated using flow cytometry analysis. Results: $P$.sarmentosum arrested the growth of $A 549$ cells at $G_{0} / G_{1}$ phase. The Annexin $V$ analysis revealed that $P$. sarmentosum exhibited significant induction of apoptosis after $24 \mathrm{~h}$ exposure. Caspases analysis showed that $P$. sarmentosum induced apoptosis through mitochondrial pathway, via the activation of caspase 3 and caspase 9. Meanwhile, cytochrome $\mathrm{c}$ analysis revealed that $P$. sarmentosum induced a mitochondrial pathway of cell death through the release of cytochrome c. Conclusions: Based on these preliminary findings, $P$. sarmentosum has a great potential as a dietary cancer treatment for lung cancer and may perhaps be used for lung cancer pharmacotherapy in the clinical settings in future.

KEYWORDS: nutritional cancer therapy, apoptosis, cancer, cell cycle 